\title{
Quantum Graphs: A simple model for Chaotic
}

\section{Scattering}

\author{
Tsampikos Kottos ${ }^{1}$ 年 Uzy Smilansky $^{2}$ \\ 1 Max-Planck-Institut für Strömungsforschung, 37073 \\ Göttingen, Germany, \\ ${ }^{2}$ Department of Physics of Complex Systems, The Weizmann \\ Institute of Science, 76100 Rehovot, Israel
}

\begin{abstract}
We connect quantum graphs with infinite leads, and turn them to scattering systems. We show that they display all the features which characterize quantum scattering systems with an underlying classical chaotic dynamics: typical poles, delay time and conductance distributions, Ericson fluctuations, and when considered statistically, the ensemble of scattering matrices reproduce quite well the predictions of appropriately defined Random Matrix ensembles. The underlying classical dynamics can be defined, and it provides important parameters which are needed for the quantum theory. In particular, we derive exact expressions for the scattering matrix, and an exact trace formula for the density of resonances, in terms of classical orbits, analogous to the semiclassical theory of chaotic scattering. We use this in order to investigate the origin of the connection between Random Matrix Theory and the underlying classical chaotic dynamics. Being an exact theory, and due to its relative simplicity, it offers new insights into this problem which is at the fore-front of the research in chaotic scattering and related fields.
\end{abstract}

submitted to J. Phys. A Special Issue - Random Matrix Theory

$\ddagger$ corresponding author: tsamp@chaos.gwdg.de 


\section{Introduction}

Quantum graphs of one-dimensional wires connected at nodes were introduced already more than half a century ago to model physical systems. Depending on the envisaged application the precise formulation of the models can be quite diverse and ranges from solid-state applications to mathematical physics [1, 2, 3, 4, 5, 6, 7, 8, 9, 10, 11, 12]. Lately, quantum graphs attracted also the interest of the quantum chaos community because they can be viewed as typical and yet relatively simple examples for the large class of systems in which classically chaotic dynamics implies universal correlations in

the semiclassical limit [13, 14, 15, 16, 17, 18, 19, 20, 21]. Up to now we have only a limited understanding of the reasons for this universality, and quantum graph models provide a valuable opportunity for mathematically rigorous investigations of the phenomenon. In particular, for quantum graphs an exact trace formula exists [12, 13, 14] which is based on the periodic orbits of a mixing classical dynamical system. Moreover, it is possible to express two-point spectral correlation functions in terms of purely combinatorial problems [16, 17, 18, 19].

By attaching infinite leads at the vertices, we get non-compact graphs, for which a scattering theory can be constructed [22, 23, 24]. They display many of the feature that characterize scattering systems with an underlying chaotic classical dynamics 25, 26, 27, 28, 29, and they are the subject of the present paper. The quantum scattering matrix for such problems can be written explicitly, together with a trace formula for the density of its resonances. These expressions are the analogues of the corresponding semiclassical approximations available in the theory of chaotic scattering [25, 26, 27], albeit here they are exact. With these tools we analyze the distribution of resonances, partial delay times, and the statistics of the fluctuating scattering amplitudes and cross sections. Moreover, we address issues like the statistical properties of the ensemble of the scattering matrices, and conductance distribution. Finally we analyze the effect of non-uniform connectivity on the statistical properties of the scattering matrix. A main part of our analysis will be focused on the comparison of the statistical properties of the above quantities with the Random Matrix Theory (RMT) predictions 30, 31, 32, 33, 34, 35, 36, 37, 38, 39, 40.

The paper is structured in the following way. In section (2), the mathematical model is introduced and the main definitions are given. Section (3) is devoted to the derivation of the scattering matrix for graphs. The trace formula for the density of resonances of quantum graphs is presented in section (4) which includes also the analysis of the underlying classical system. The next section, is dedicated to the analysis of various statistical properties of the $S$-matrix. Our numerical data are compared both with the predictions of RMT and with the semiclassical expectations. Finally, in section (6), we analyze the class of star-graphs for which several results can be analytically derived. Our conclusions are summarized in section (7). 


\section{Quantum Graphs: Definitions}

We start by considering a compact graph $\mathcal{G}$. It consists of $V$ vertices connected by $B$ bonds. The number of bonds which emanate from each vertex $i$ defines the valency $v_{i}$ of the corresponding vertex (for simplicity we will allow only a single bond between any two vertices). The graph is called $v$-regular if all the vertices have the same valency $v$. The total number of bonds is $B=\frac{1}{2} \sum_{i=1}^{V} v_{i}$. Associated to every graph is its connectivity matrix $C$. It is a square matrix of size $V$ whose matrix elements $C_{i, j}$ take the values 1 if the vertices $i, j$ are connected with a bond, or 0 otherwise. The bond connecting the vertices $i$ and $j$ is denoted by $b \equiv(i, j)$, and we use the convention that $i<j$. It will be sometimes convenient to use the "time reversed" notation, where the first index is the larger, and $\hat{b} \equiv(j, i)$ with $j>i$. We shall also use the directed bonds representation, in which $b$ and $\hat{b}$ are distinguished as two directed bonds conjugated by time-reversal. We associate the natural metric to the bonds, so that $x_{i, j}\left(x_{j, i}\right)$ measures the distance from the vertex $i(j)$ along the bond. The length of the bonds are denoted by $L_{b}$ and we shall henceforth assume that they are rationally independent. The mean length is defined by $\langle L\rangle \equiv(1 / B) \sum_{b=1}^{B} L_{b}$ and in all numerical calculations bellow it will be taken to be 1 . In the directed- bond notation $L_{b}=L_{\hat{b}}$.

The scattering graph $\tilde{\mathcal{G}}$ is obtained by adding leads which extend from $M(\leq V)$ vertices to infinity. For simplicity we connect at most one lead to any vertex. The valency of these vertices increases to $\tilde{v}_{i}=v_{i}+1$. The $M$ leads are denoted by the index $i$ of the vertex to which they are attached while $x_{i}$ now measures the distance from the vertex along the lead $i$.

The Schrödinger operator (with $\hbar=2 m=1$ ) is defined on the graph $\tilde{\mathcal{G}}$ in the following way: On the bonds $b$, the components $\Psi_{b}$ of the total wave function $\Psi$ are solutions of the one - dimensional equation

$$
\left(-i \frac{\mathrm{d}}{\mathrm{d} x}-A_{b}\right)^{2} \Psi_{b}(x)=k^{2} \Psi_{b}(x), \quad b=(i, j)
$$

where $A_{b}$ (with $\Re e\left(A_{b}\right) \neq 0$ and $A_{b}=-A_{\hat{b}}$ ) is a "magnetic vector potential" which breaks the time reversal symmetry. In most applications we shall assume that all the $A_{b}$ 's are equal and the bond index will be dropped. The components of the wave functions on the leads, $\Psi_{i}(x)$, are solutions of

$$
-\frac{\mathrm{d}^{2}}{\mathrm{~d} x^{2}} \Psi_{i}(x)=k^{2} \Psi_{i}(x), \quad i=1, \ldots, M
$$

At the vertices, the wavefunction satisfies boundary conditions which ensure current conservation. To implement the boundary conditions, the components of the wave function on each of the bonds $b$ and the leads $i$ are expressed in terms of counter propagating waves with a wave-vector $k$ :

On the bonds : $\Psi_{b}=a_{b} \mathrm{e}^{i\left(k+A_{b}\right) x_{b}}+c_{b} \mathrm{e}^{i\left(-k+A_{b}\right) x_{b}}$
On the leads : $\Psi_{i}=I_{i} \mathrm{e}^{-i k x_{i}}+O_{i} \mathrm{e}^{i k x_{i}}$ 
The amplitudes $a_{b}, c_{b}$ on the bonds and $I_{i}, O_{i}$ on the lead are related by

$$
\left(\begin{array}{c}
O_{i} \\
a_{i, j_{1}} \\
\cdot \\
a_{i, j_{v_{i}}}
\end{array}\right)=\Sigma^{(i)}\left(\begin{array}{c}
I_{i} \\
c_{j_{1}, i} \\
\cdot \\
c_{j_{v_{i}}, i}
\end{array}\right), \quad \Sigma^{(i)}=\left(\begin{array}{cccc}
\rho^{(i)} & \tau_{j_{1}}^{(i)} & \cdot & \tau_{j_{v_{i}}}^{(i)} \\
\tau_{j_{1}}^{(i)} & \tilde{\sigma}_{j_{1}, j_{1}}^{(i)} & \cdot & \tilde{\sigma}_{j_{1}, j_{v_{i}}}^{(i)} \\
\cdot & \cdot & \cdot & \cdot \\
\tau_{j_{v_{i}}}^{(i)} & \tilde{\sigma}_{j_{v_{i}, j_{1}}^{(i)}}^{(i)} & \cdot \tilde{\sigma}_{j_{v_{i}}, j_{v_{i}}}^{(i)}
\end{array}\right) .
$$

These equalities impose the boundary conditions at the vertices. The vertex scattering matrices $\Sigma_{j, j^{\prime}}^{(i)}$, are $\tilde{v}_{i} \times \tilde{v}_{i}$ unitary symmetric matrices, and $j, j^{\prime}$ go over all the $v_{i}$ bonds and the lead which emanate from $i$. The unitarity of $\Sigma^{(i)}$ guarantees current conservation at each vertex.

On the right hand side of (田), the vertex scattering matrix $\Sigma^{(i)}$ was written explicitly in terms of the vertex reflection amplitude $\rho^{(i)}$, the lead-bond transmission amplitudes $\left\{\tau_{j}^{(i)}\right\}$, and the $v_{i} \times v_{i}$ bond-bond transition matrix $\tilde{\sigma}_{j, j^{\prime}}^{(i)}$, which is sub unitary $\left(\left|\operatorname{det} \tilde{\sigma}^{(i)}\right|<1\right.$ ), due to the coupling to the leads.

Graphs for which there are no further requirements on the $\Sigma^{(i)}$ shall be referred to as generic. It is often convenient to compute the vertex scattering matrix from a requirement that the wave function is continuous and satisfies Neumann boundary conditions at that vertex. These graphs shall be referred to as Neumann graphs, and the resulting $\tilde{\Sigma}^{(i)}$ matrices read:

$$
\tilde{\sigma}_{j, j^{\prime}}^{(i)}=\frac{2}{\tilde{v}}-\delta_{j, j^{\prime}} ; \quad \tau_{j}^{(i)}=\frac{2}{\tilde{v}} ; \quad \rho^{(i)}=\frac{2}{\tilde{v}}-1 .
$$

Vertices which are not coupled to leads have $\rho^{i}=1, \tau_{j}^{(i)}=0$, while the bond-bond transition matrix $\tilde{\sigma}_{j \cdot j^{\prime}}^{(i)}=\frac{2}{v}-\delta_{j, j^{\prime}}$ is unitary.

\section{The S-matrix for Quantum Graphs}

It is convenient to discuss first graphs with leads connected to all the vertices $M=$ $V$. The generalization to an arbitrarily number $M \leq V$ of leads (channels) is straightforward and will be presented at the end of this section.

To derive the scattering matrix, we first write the bond wave functions using the two representations which are conjugated by "time reversal":

$$
\begin{aligned}
\Psi_{b}\left(x_{b}\right) & =a_{b} \mathrm{e}^{i\left(k+A_{b}\right) x_{b}}+c_{b} \mathrm{e}^{i\left(-k+A_{b}\right) x_{b}}= \\
\Psi_{\hat{b}}\left(x_{\hat{b}}\right) & =a_{\hat{b}} \mathrm{e}^{i\left(k+A_{\hat{b}}\right) x_{\hat{b}}}+c_{\hat{i}} \mathrm{e}^{i\left(-k+A_{\hat{b}}\right) x_{\hat{b}}}= \\
& =a_{\hat{b}} \mathrm{e}^{i\left(-k-A_{\hat{b}}\right) x_{b}} \mathrm{e}^{i\left(k+A_{\hat{b}}\right) L_{b}}+c_{\hat{b}} \mathrm{e}^{i\left(-k+A_{\hat{b}}\right) L_{b}} \mathrm{e}^{i\left(k-A_{\hat{b}}\right) x_{b}} .
\end{aligned}
$$

Hence,

$$
c_{b}=a_{\hat{b}} \mathrm{e}^{i\left(k+A_{\hat{b}}\right) L_{b}}, \quad a_{b}=c_{\hat{b}} \mathrm{e}^{i\left(-k+A_{\hat{b}}\right) L_{b}} .
$$

In other words, but for a phase factor, the outgoing wave from the vertex $i$ in the direction $j$ is identical to the incoming wave at $j$ coming from $i$. 
Substituting $a_{b}$ from Eq. (7) in Eq. (অ), and solving for $c_{i, j}$ we get

$$
\begin{aligned}
& c_{i, j^{\prime}}=\sum_{r, s}\left(\mathbf{1}-\tilde{S}_{B}(k ; A)\right)_{(i, r),(s, j)}^{-1} D_{(s, j)} \tau_{s}^{(j)} I_{j} \\
& O_{i}=\rho^{(i)} I_{i}+\sum_{j^{\prime}} \tau_{j^{\prime}}^{(i)} c_{i j^{\prime}}
\end{aligned}
$$

where 1 is the $2 B \times 2 B$ unit matrix. Here, the "bond scattering matrix" $\tilde{S}_{b}$ is a subunitary matrix in the $2 B$ dimensional space of directed bonds which propagates the wavefunctions. It is defined as $\tilde{S}_{B}(k, A)=D(k ; A) \tilde{R}$, with

$$
\begin{aligned}
D_{i j, i^{\prime} j^{\prime}}(k, A) & =\delta_{i, i^{\prime}} \delta_{j, j^{\prime}} \mathrm{e}^{i k L_{i j}+i A_{i, j} L_{i j}} \\
\tilde{R}_{j i, n m} & =\delta_{n, i} C_{j, i} C_{i, m} \tilde{\sigma}_{j i, i m}^{(i)} .
\end{aligned}
$$

$D(k, A)$ is a diagonal unitary matrix which depends only on the metric properties of the graph, and provides a phase which is due to free propagation on the bonds. The sub-unitary matrix $\tilde{R}$ depends on the connectivity and on the bond-bond transition matrices $\tilde{\sigma}$. It assigns a scattering amplitude for transitions between connected directed bonds. $\tilde{R}$ is sub-unitary, since

$$
|\operatorname{det} \tilde{R}|=\prod_{i=1}^{V}\left|\operatorname{det} \tilde{\sigma}^{(i)}\right|<1 \text {. }
$$

Replacing $c_{i, j^{\prime}}$ in the second of Eqs. (8) we get the following relation between the outgoing and incoming amplitudes $O_{i}$ and $I_{j}$ on the leads:

$$
O_{i}=\rho^{(i)} I_{i}+\sum_{j^{\prime} j r s} \tau_{j^{\prime}}^{(i)}\left(\mathbf{1}-\tilde{S}_{B}(k ; A)\right)_{(i, r),(s, j)}^{-1} D_{(s, j)} \tau_{s}^{(j)} I_{j}
$$

Combining (11) for all leads $i=1, \ldots, V$, we obtain the unitary $V \times V$ scattering matrix $S^{(V)}$

$$
S_{i, j}^{(V)}=\delta_{i, j} \rho^{(i)}+\sum_{r, s} \tau_{r}^{(i)}\left(\mathbf{1}-\tilde{S}_{B}(k ; A)\right)_{(i, r),(s, j)}^{-1} D_{(s, j)} \tau_{s}^{(j)} .
$$

From Eq. (12), we see that the scattering matrix may be decomposed into two parts $S^{(V)}(k)=S^{D}+S^{f l}(k)$ which are associated with two well separated time scales of the scattering process. $S^{D}\left(=\delta_{i, j} \rho^{i}\right)$ is the prompt reflection at the entrance vertex and induces a "direct" component. In general, it varies very slowly with energy, and is system dependent. On the other hand the "chaotic" component of the $S$ matrix, $S^{f l}(k)$, starts by a transmission from the incoming lead $i$ to the bonds $(i, r)$ with transmission amplitudes $\tau_{r}^{(i)}$. The wave gains a phase $\mathrm{e}^{i\left(k+A_{b}\right) L_{b}}$ for each bond it traverses and a scattering amplitude $\tilde{\sigma}_{r, s}^{(i)}$ at each vertex. This multiple scattering inside the interaction region becomes apparent when the expansion

$$
\left(\mathbf{1}-\tilde{S}_{B}(k ; A)\right)^{-1}=\sum_{n=0}^{\infty} \tilde{S}_{B}^{n}(k ; A)
$$


is substituted in (12). Eventually the wave is transmitted from the bond $(s, j)$ to the lead $j$ with an amplitude $\tau_{s}^{(j)}$. Explicitly,

$$
S_{i, j}^{(V)}=\delta_{i, j} \rho^{(i)}+\sum_{t \in \mathcal{T}_{i \rightarrow j}} \mathcal{B}_{t} \mathrm{e}^{i\left(k l_{t}+\Theta_{t}\right)}
$$

where $\mathcal{T}_{i \rightarrow j}$ is the set of the trajectories on $\tilde{\mathcal{G}}$ which lead from $i$ to $j . \mathcal{B}_{t}$ is the amplitude corresponding to a path $t$ whose length and directed length are $l_{t}=\sum_{b \in t} L_{b}$ and $\Theta_{t}=\sum_{b \in t} L_{b} A_{b}$ respectively. Thus the scattering amplitude $S_{i, j}^{(V)}$ is a sum of a large number of partial amplitudes, whose complex interference brings about the typical irregular fluctuations of $\left|S_{i, j}^{(V)}\right|^{2}$ as a function of $k$.

One of the basic concepts in the quantum theory of scattering are the resonances. They represent long-lived intermediate states to which bound states of a closed system are converted due to coupling to continua. On a formal level, resonances show up as poles of the scattering matrix $S^{(M)}$ occurring at complex wave-numbers $\kappa_{n}=k_{n}-\frac{i}{2} \Gamma_{n}$, where $k_{n}$ and $\Gamma_{n}$ are the position and the width of the resonances, respectively. From (12) it follows that the resonances are the complex zeros of

$$
\zeta_{\tilde{\mathcal{G}}}(\kappa)=\operatorname{det}\left(1-\tilde{S}_{B}(\kappa ; A)\right)=0 .
$$

The eigenvalues of $\tilde{S}_{B}$ are in the unit circle, and therefore the resonances appear in the lower half of the complex $\kappa$ plane. Moreover from Eq. (15) it is clear that their formation is closely related to the internal dynamics inside the scattering region which is governed by $\tilde{S}_{B}$.

There exists an intimate link between the scattering matrix and the spectrum of the corresponding closed graph. It manifests the exterior -interior duality [41] for graphs. The spectrum of the closed graph is the set of wave-numbers for which $S^{(V)}$ has +1 as an eigenvalue. This corresponds to a solution where no currents flow in the leads so that the conservation of current is satisfied on the internal bonds. 1 is in the spectrum of $S^{(V)}$ if

$$
\zeta_{\mathcal{G}}(k)=\operatorname{det}\left[\mathbf{1}-S^{(V)}(k)\right]=0
$$

Eq. (16) can be transformed in an alternative form

$$
\zeta_{\mathcal{G}}(k)=\operatorname{det}[\mathbf{1}-\rho] \frac{\operatorname{det}[\mathbf{1}-D(k) R]}{\operatorname{det}[\mathbf{1}-D(k) \tilde{R}]}=0 \quad ; \quad R_{i, r ; s, j}=\tilde{R}_{i, r ; s, j}+\delta_{r, s} \frac{\tau_{i}^{(r)} \tau_{j}^{(r)}}{1-\rho^{(r)}}
$$

which is satisfied once

$$
\operatorname{det}[\mathbf{1}-D(k) R]=0 .
$$

In contrast to $\tilde{R}, R$ is a unitary matrix in the space of directed bonds, and therefore the spectrum is real. (18) is the secular equation for the spectrum of the compact part of the graph, and it was derived in a different way in [13.

The difference $\delta R=R-\tilde{R}$ gets smaller as larger graphs are considered (for graphs with Neumann boundary conditions it is easy to see that the difference is of order $\frac{1}{v}$ ). That is, the leads are weekly coupled to the compact part of the graph, and one can use 

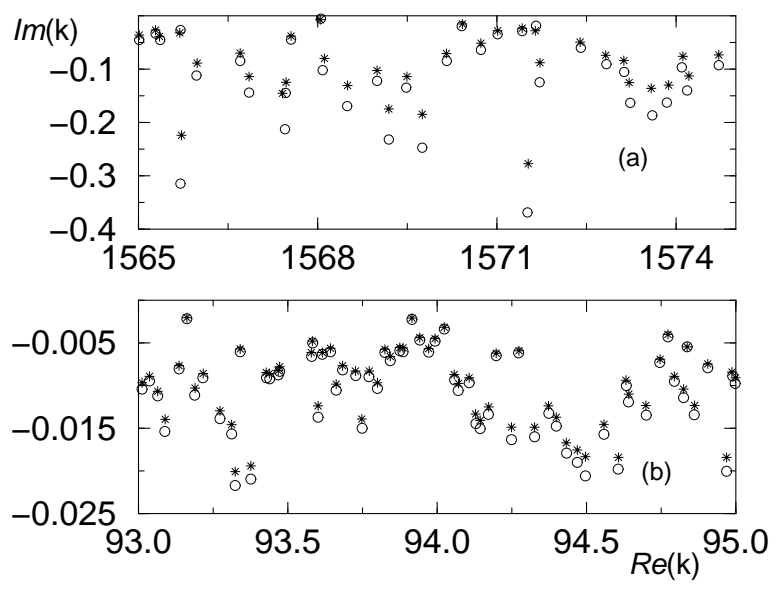

Figure 1. Poles of the $S^{(V)}$-matrix for regular Neumann graphs. The exact evaluated poles are indicated with $(\circ)$ while $(\star)$ are the results of the perturbation theory (20): (a) $V=5$ and $v=4$ and (b) $V=15$ and $v=14$.

perturbation theory for the computation of the resonance parameters. To lowest order, $(\delta R=0)$, the resonances coincide with the spectrum of the compact graph. Let $k_{n}$ be in the spectrum. Hence, there exists a vector $|n\rangle$ which satisfies the equation

$$
D\left(k_{n}\right) R|n\rangle=1|n\rangle .
$$

To first order in $\delta R$, the resonances acquire a width

$$
\delta \kappa_{n}=-i \frac{\left\langle n\left|D\left(k_{n}\right) \delta R\right| n\right\rangle}{\langle n|L| n\rangle} .
$$

To check the usefulness of this result, we searched numerically for the true poles for a few scattering graphs and compared them with the approximation (20). In figure 1 we show the comparison for fully connected Neumann graphs with $V=5,15$ and $A=0$. As expected the agreement between the exact poles and the perturbative results improves as $v$ increases.

We finally comment that the formalism above can be easily modified for graphs where not all the vertices are attached to leads. If the vertex $l$ is not attached, one has to set $\rho^{(l)}=1, \tau_{j}^{(l)}=0$ in the definition of $\Sigma^{(l)}$. The dimension of the scattering matrix is then changed accordingly.

For the sake of completeness we quote here an alternative expression for the $S$ matrix which applies for Neumann graphs, exclusively [14. For this purpose we define the $V \times V$ matrix

$$
h_{i, j}(k, A)=\left\{\begin{array}{cc}
-\sum_{m \neq i} C_{i, m} \cot \left(k L_{i, m}\right), & i=j \\
C_{i, j} \mathrm{e}^{-i A_{i, j} L_{i, j}}\left(\sin \left(k L_{i, j}\right)\right)^{-1}, & i \neq j
\end{array}\right.
$$

in terms of which,

$$
S^{(V)}=(i \mathbf{1}+h(k))^{-1}(i \mathbf{1}-h(k))
$$


where $\mathbf{1}$ is the $V \times V$ unit matrix. $S^{(V)}$ is unitary since $h(k)$ is hermitian.

In the case of graphs connected to leads at an arbitrary set of $M<V$ vertices with indices $\left\{i_{l}\right\}, \quad l=1, \cdots, M$, the $M \times M$ scattering matrix $S^{(M)}$ has to be modified in the following way

$$
S^{(M)}=2 i W\left(h(k)+i W^{T} W\right)^{-1} W^{T}-\mathbf{1} .
$$

Here $W_{i_{l}, j}=\delta_{i_{l}, j}$ is the $M \times V$ leads - vertices coupling matrix, ( $W=\mathbf{1}$ when $M=V$ ). This form of the $S$ matrix is reminiscent of the expression which was introduced by Weidenmüller to generalize the Breit-Wigner theory for many channels and internal states. However, (23) is an exact expression which involves no truncations.

It follows from (23) that in the present case, one can identify the poles of the $S$ matrix with the zeros of

$$
\zeta_{\tilde{\mathcal{G}}}(\kappa)=\operatorname{det}\left(h(\kappa)+i W^{T} W\right)=0 .
$$

Its main advantage over (15) is that it involves a determinant of a matrix of much lower dimension.

\section{The trace formula for resonances and classical scattering on graphs}

In the spectral theory of bounded hamiltonian systems, the most fundamental object of study is the spectral density which consists of a sum of $\delta$ functions at the spectral points. For open systems, it is replaced by the resonance density, which is defined on the real $k$ line and consists of an infinite sum of Lorentzians which are centered at $k_{n}=\Re e \kappa_{n}$ and have width $\Gamma_{n}=-2 \Im m \kappa_{n}$, where $\kappa_{n}$ are the complex poles of the scattering matrix. In the present section we shall express the resonance density for scattering graphs in terms of periodic orbits of their compact part. This is the analogue of the trace formula for bounded graphs.

\subsection{The Trace Formula}

The resonance density $d_{R}(k)$ can be deduced from the total phase $\Phi(k)=$ $\frac{1}{i} \ln \operatorname{det} S^{(M)}(k)$ of the scattering matrix 43$]$ :

$$
d_{R}(k) \equiv \frac{1}{2 \pi} \frac{d \Phi}{d k}=-i \frac{1}{2 \pi} \frac{\partial}{\partial k} \ln \operatorname{det} S^{(M)}(k) .
$$

It is a smooth function for real $k$, and can be interpreted as the mean length of the delay associated with the scattering at wave-number $k$ [26].

Using Eq. (12) and performing standard manipulations [42], we obtain the following expression for the phase $\Phi(k)$

$$
\Phi(k)-\Phi(0)=-2 \Im m \ln \operatorname{det}\left(I-\tilde{S}_{B}(k ; A)\right)+\mathcal{L} k .
$$

where $\mathcal{L}=2 \sum_{b=1}^{B} L_{b}$ is twice the total length of the bonds of $\tilde{\mathcal{G}}$. 
Using the expansion

$$
\ln \operatorname{det}\left(I-\tilde{S}_{B}(k ; A)\right)=-\sum_{n=1}^{\infty} \frac{1}{n} \operatorname{tr} \tilde{S}_{B}^{n}(k ; A)
$$

we rewrite Eq. (26) as

$$
\Phi(k)=\Phi(0)+\mathcal{L} k+2 \Im m \sum_{n=1}^{\infty} \frac{1}{n} \operatorname{tr} \tilde{S}_{B}^{n}(k ; A) .
$$

On the other hand, using Eq. (9) we can write $\operatorname{tr} \tilde{S}_{B}^{n}(k ; A)$ as sums over $n$-periodic orbits on the graph

$$
\operatorname{tr}\left(\tilde{S}_{B}^{n}(k ; A)\right)=\sum_{p \in \mathcal{P}_{n}} n_{p} \tilde{\mathcal{A}}_{p}^{r} \mathrm{e}^{i\left(l_{p} k+\Theta_{p}\right) r},
$$

where the sum is over the set $\mathcal{P}_{n}$ of primitive periodic orbits whose period $n_{p}$ is a divisor of $n$, with $r=n / n_{p}$ (primitive periodic orbits are those which cannot be written as a repetition of a shorter periodic orbit). The amplitudes $\tilde{\mathcal{A}}_{p}$ are the products of the bond-bond scattering amplitudes $\tilde{\sigma}_{b, b^{\prime}}^{(i)}$ along the primitive loops i.e.

$$
\tilde{\mathcal{A}}_{p}=\prod_{i=1}^{n_{p}} \tilde{\sigma}_{b, b^{\prime}}^{(i)} .
$$

Substituting Eqs. $(28,29)$ in Eq. (25) one gets the resonance density

$$
d_{R}(k)=\frac{1}{2 \pi} \mathcal{L}+\frac{1}{\pi} \Re e \sum_{n=1}^{\infty} \sum_{p \in \mathcal{P}_{n}} n_{p} l_{p} r \tilde{\mathcal{A}}_{p}^{r} \mathrm{e}^{i\left(l_{p} k+\Theta_{p}\right) r}
$$

Eq. (31) is an exact trace formula for the resonance density. The first term on the right hand side of Eq. (31) corresponds to the smooth resonance density, while the second provides the fluctuating part. We notice that the mean resonance spacing is given by

$$
\Delta=\frac{2 \pi}{\mathcal{L}} \simeq \frac{2 \pi}{2 B\langle L\rangle}
$$

and it is the same as the mean level spacing obtained for the corresponding bounded graph $\mathcal{G}[13]$.

\subsection{Classical Dynamics}

We conclude this section with the discussion of the classical dynamics on the graph $\tilde{\mathcal{G}}$. A classical particle moves freely as long as it is on a bond. The vertices are singular points, and it is not possible to write down the analogue of the Newton equations there. In [13, 14] it was shown that it is possible to define a classical evolution on the graph: A Poincaré section on the graph consists of the discrete set of directed bonds. The phase-space density at a (topological) time $n$ is the set of occupation probabilities $\rho_{b}(n)$ of the directed bonds, and the classical evolution is governed by a Markovian master equation. Applied to the compact part of a scattering graph it reads,

$$
\tilde{\rho}_{b}(n+1)=\sum_{b^{\prime}} \tilde{U}_{b, b^{\prime}} \tilde{\rho}_{b^{\prime}}(n)
$$


where the transition matrix $\tilde{U}_{b, b^{\prime}}$ is given by the corresponding quantum transition probability

$$
\tilde{U}_{i j, n m}=\left|\tilde{R}_{i j, n m}\right|^{2}=\delta_{j, n}\left|\sigma_{i j, j m}^{(j)}\right|^{2} .
$$

Notice that $\tilde{U}$ does not involve any metric information on the graph.

Due to loss of flux to the leads $\sum_{b^{\prime}} \tilde{U}_{b b^{\prime}}<1$, and the phase-space measure is not preserved, but rather, decays in time. The probability to remain on $\tilde{\mathcal{G}}$ is

$$
\tilde{P}(n) \equiv \sum_{b=1}^{2 B} \rho_{b}(n)=\sum_{b, b^{\prime}} \tilde{U}_{b b^{\prime}} \rho_{b^{\prime}}(n-1) \simeq \mathrm{e}^{-\Gamma_{c l} n} \tilde{P}(0)
$$

where $\exp \left(-\Gamma_{c l}\right)$ is the largest eigenvalue of the "leaky" evolution operator $\tilde{U}_{b b^{\prime}}$.

For the $v$-regular graph with $\tilde{\sigma}^{(i)}$ given by (5) the spectrum of $\tilde{U}$ is restricted to the interior of a circle with radius given by the maximum eigenvalue $\nu_{1}=(v-1) \tau^{2}+\rho^{2}$ with corresponding eigenvector $\mid 1>=(1 / 2 B)(1,1, \cdots, 1)^{T}$. Hence the decay rate $\Gamma_{c l}=-\ln \nu_{1}$ for regular Neumann graphs take the simple form

$$
\Gamma_{c l}=-\ln \left(1-\tau^{2}\right) \approx(2 /(1+v))^{2} .
$$

We notice that removing the leads from the vertices and turning $\tilde{\mathcal{G}}$ into a compact graph $\mathcal{G}$ we get $\Gamma_{c l}=0$ since in this case $\tau^{(i)}=0$ (and $\rho^{(i)}=1$ ) and the phase-space measure is preserved as expected.

The inverse decay rate $T_{c l}=\Gamma_{c l}^{-1}$, gives the average classical delay time that the particle spends within the interaction region. Injecting a particle from the leads to the scattering domain, its probability to be on any bond randomizes, because at each vertex a Markovian choice of one out of $v$ directions is made. The longer a particle remains within the interaction regime, the more scattering events it experiences. The set of trapped trajectories whose occupancy decays exponentially in time is the analogue of the strange repeller in generic Hamiltonian systems displaying "chaotic scattering".

\section{Statistical Analysis of the $S$-matrix}

So far we developed the scattering theory of graphs, pointing out their similarity with scattering systems which display chaotic scattering in the classical limit. Due to the interference of a large number of amplitudes, the $S$-matrix fluctuates as a function of $k$, and its further analysis calls for a statistical approach which will be the subject of the present section. We shall show that quantum graphs possess typical poles, delay time and conductance distributions, Ericson fluctuations of the scattering amplitudes, and when considered statistically, the ensemble of scattering matrices are very well reproduced by the predictions of RMT. At the same time deviations from the universal RMT results, which are related to the system- specific properties of some graphs, will be pointed out. The study of these deviations is especially convenient for graphs because of the transparent and simple scattering theory developed in terms of scattering trajectories. 


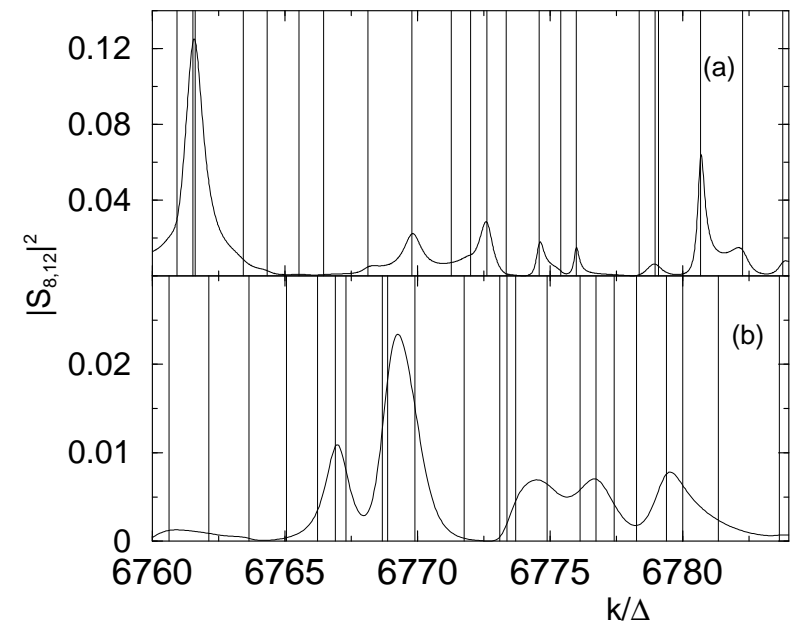

Figure 2. Representative examples of scattering cross sections $\left|S_{i, j}^{(V)}\right|^{2}$ for Neumann graphs in a regime of (a) isolated resonances $(V=15, v=14$ with corresponding $\left.\langle\gamma\rangle_{k}=0.59\right)$ and (b) overlapping resonances $(V=49, v=14$ with corresponding $\langle\gamma\rangle_{k} \simeq 2$ ). The real parts of the resonances in this energy interval are indicated by the vertical lines.

An important parameter which is associated with the statistical properties of the $S$-matrix is the Ericson parameter defined through the scaled mean resonance width as:

$$
\langle\gamma\rangle_{k} \equiv \frac{\left\langle\Gamma_{n}\right\rangle_{k}}{\Delta}
$$

where $\langle\cdot\rangle_{k}$ denotes spectral averaging and $\Delta$ is the mean spacing between resonances. The Ericson parameter determines whether the resonances overlap $\left(\langle\gamma\rangle_{k}>1\right)$ or are isolated $\left(\langle\gamma\rangle_{k}<1\right)$. Typical example for the two extreme situations are shown in figure 2.

The degree of resonance overlap determines the statistical properties of the $S$ matrix. We shall show in the sequel that the mean width can be approximated by the classical decay rate $\langle\gamma\rangle_{k}=\gamma_{c l}$. For the $v$ regular graphs discussed above, we have

$$
\gamma_{c l} \equiv \frac{\Gamma_{c l}}{\Delta} \approx \frac{4}{2 \pi} \frac{v}{1+v} \frac{V}{1+v}
$$

where we made use of Eqs. (32, 36). Thus changing $v$ and $V$ we can control the degree of overlap allowing to test various phenomena.

In what follows, unless explicitly specified, we shall consider regular graphs with one lead attached to each vertex, i.e. $M=V$. Finally, the widths are always scaled by the mean spacing $\Delta$ i.e. $\gamma_{n} \equiv \frac{\Gamma_{n}}{\Delta}$. 


\subsection{The resonance width distribution}

The resonance width distribution can be computed for a given graph in the following way. Consider the complex $\kappa=x+i y$ plane, where the zeros of the secular function

$$
\zeta_{\tilde{\mathcal{G}}}(\kappa)=\operatorname{det}\left(\mathbf{1}-\tilde{S}_{B}(\kappa ; A)\right)=f(x, y)+i g(x, y) .
$$

are the poles of the $S$ matrix (resonances). The variables $(x, y)$ are expressed in units of $\Delta$. On average, the number of resonances with real part, $x \in[0, X]$ is $X$. The density of resonance widths reads

$$
\mathcal{P}(\gamma)=\lim _{X \rightarrow \infty} \frac{1}{X} \int_{0}^{X} \delta(f(x, y=-\gamma)) \delta(g(x, y=-\gamma))\left|\frac{\mathrm{d} \zeta_{\tilde{\mathcal{G}}}(\kappa)}{\mathrm{d} \kappa}\right|_{y=-\gamma}^{2} \mathrm{~d} x
$$

We used the Cauchy-Riemann theorem for the evaluation of the Jacobian $\left(f_{x} g_{y}-f_{y} g_{x}\right)$ which multiplies the two $\delta$ functions that locate the complex zeros of $\zeta_{\tilde{\mathcal{G}}}$. We now recall that the $\kappa$ dependence of $\tilde{S}_{B}(\kappa ; A)$ comes from the factors $\mathrm{e}^{i \kappa L_{b}}$ in (9). This implies that for a given $y, \zeta_{\tilde{\mathcal{G}}}$ is a quasi periodic function of $x$. Moreover, expanding the determinant (39) it is not difficult to show that the frequencies involved can be written as linear combinations of the bond lengths $\lambda=\sum q_{b} L_{b}$ with integer coefficients $q_{b}=0$, 1 , or 2 . Since the bond lengths are rationally independent, we find that $\zeta_{\tilde{\mathcal{G}}}$ depends on a finite number of incommensurate frequencies. The expression (40) can be regarded as a "time" integral over a trajectory on a multidimensional incommensurate torus, which covers the torus ergodically. Hence, the integral can be replaced by a phase space average,

$$
\mathcal{P}(\gamma)=\int_{0}^{2 \pi} \frac{\mathrm{d} \psi_{1}}{2 \pi} \cdots \int_{0}^{2 \pi} \frac{\mathrm{d} \psi_{J}}{2 \pi} \delta(f(\vec{\psi},-\gamma)) \delta(g(\vec{\psi},-\gamma))\left|\frac{\mathrm{d} \zeta_{\tilde{\mathcal{G}}}(\vec{\psi},-\gamma)}{\mathrm{d} \kappa}\right|^{2}
$$

Here $\vec{\psi}$ denotes the vector of independent angles on the $J$ dimensional torus. Although the above formula provides a general framework, its application to actual graphs is a formidable task.

An important feature of the distribution of the resonances in the complex plane can be deduced by studying the secular function $\zeta_{\tilde{\mathcal{G}}}(\kappa)$. Consider $\zeta_{\tilde{\mathcal{G}}}(\kappa=0)$. If one of the eigenvalues of the matrix $\tilde{R}(9)$ takes the value $1, \zeta_{\tilde{\mathcal{G}}}(k=0)=0$ and because of the quasi-periodicity of $\zeta_{\tilde{\mathcal{G}}}$, its zeros reach any vicinity of the real axis infinitely many times. The largest eigenvalue of the $\tilde{R}$ matrix for $v$-regular Neumann graphs is 1 , and therefore the distribution of resonance widths is finite in the vicinity of $\gamma=0$. For generic graphs, the spectrum of $\tilde{R}$ is inside a circle of radius $\lambda_{\max }<1$. This implies that the poles are excluded from a strip just under the real axis, whose width can be estimated by

$$
\Gamma_{g a p}=-2 \ln \left(\left|\lambda_{\max }\right|\right) / L_{\max }
$$

where $L_{\max }$ is the maximum bond length. The existence of a gap is an important feature of the resonance width distribution $\mathcal{P}(\gamma)$ for chaotic scattering systems.

A similar argument was used recently in 23] in order to obtain an upper bound for the resonance widths. It is

$$
\Gamma_{\max } \sim-2 \ln \left(\left|\lambda_{\min }\right|\right) / L_{\min },
$$




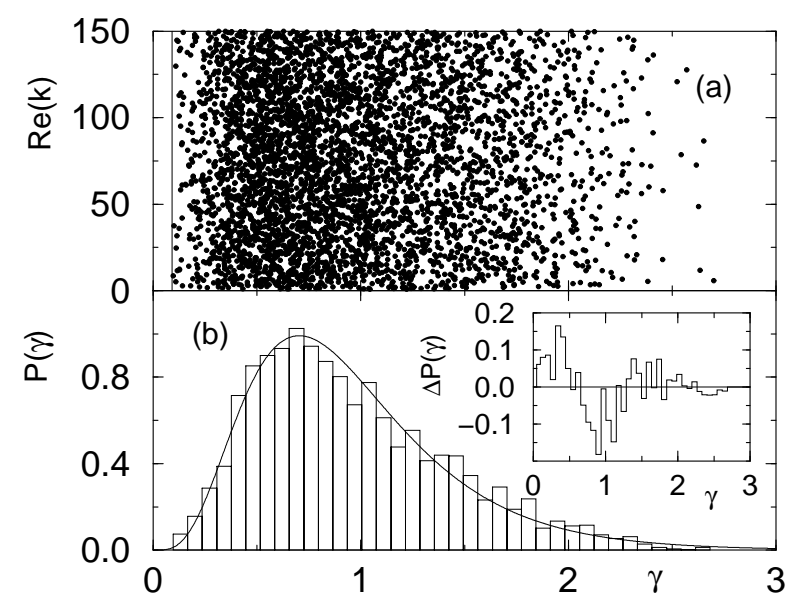

Figure 3. (a) The 5000 resonances of a single realization of a complete $V=5$ graph with $A \neq 0$ and $M=V$. The solid line marks the position of the gap $\gamma_{g a p}$. (b) The distribution of resonance widths $\mathcal{P}(\gamma)$. The solid line is the RMT prediction (45). The difference $\mathcal{P}(\gamma)-\mathcal{P}_{C U E}(\gamma)$ is shown in the inset.

where $\lambda_{\min }$ and $L_{\min }$ are the minimum eigenvalue and bond length, respectively.

The distribution of the complex poles for a generic fully connected graph with $V=5$ is shown in figure $3 \mathrm{a}$. The vertical line which marks the region from which resonances are excluded was computed using (42).

Random matrix theory can provide an expression for the distribution of resonances. In the case of non-overlapping resonances, perturbation theory shows that the resonance widths are distributed according to the so-called $\chi^{2}$ distribution

$$
\mathcal{P}(\gamma)=\frac{(\beta M / 2)^{\beta M / 2}}{\langle\gamma\rangle_{k} \Gamma(\beta M / 2)}\left(\frac{\gamma}{\langle\gamma\rangle_{k}}\right)^{\beta M / 2-1} \exp \left(-\gamma \beta M / 2\langle\gamma\rangle_{k}\right),
$$

where $\beta=1(2)$ for systems which respect (break) time-reversal symmetry, and $\Gamma(x)$ is the gamma-function. Once $\langle\gamma\rangle_{k}$ becomes large enough the resonances start to overlap, and (44) does not hold. In the general case, Fyodorov and Sommers 36, 37] proved that the distribution of scaled resonance widths for the unitary random matrix ensemble, is given by

$$
\mathcal{P}(\gamma)=\frac{(-1)^{M}}{\Gamma(M)} \gamma^{M-1} \frac{d^{M}}{d \gamma^{M}}\left(\mathrm{e}^{-\gamma \pi g} \frac{\sinh (\gamma \pi)}{(\gamma \pi)}\right)
$$

where the parameter $g=\frac{2}{\left(1-\left\langle S^{D}\right\rangle_{k}^{2}\right)}-1$ controls the degree of coupling with the channels (and is assumed that $\left.g_{i}=g \forall i=1, \ldots M\right)$. For $g \gg 1$ (i.e. weak coupling regime) Eq. (45) reduces to (44).

In the limit of $M \gg 1$, Eq. (45) reduces to the following expression 37

$$
\mathcal{P}(\gamma)=\left\{\begin{array}{ccc}
\frac{M}{2 \pi \gamma^{2}} & \text { for } & \frac{M}{\pi(g+1)}<\gamma<\frac{M}{\pi(g-1)} \\
0 & \text { otherwise }
\end{array} .\right.
$$




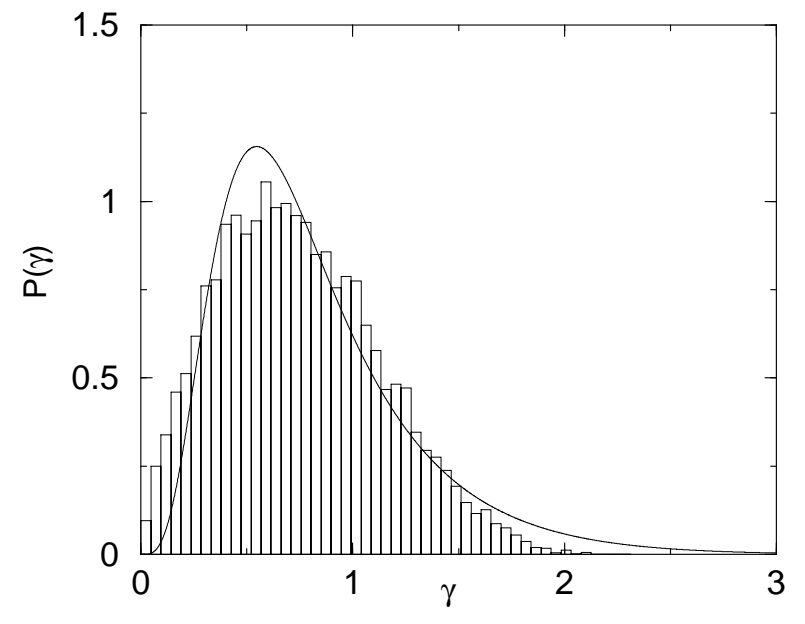

Figure 4. Resonance width distribution $\mathcal{P}(\gamma)$ for a complete Neumann graph with $M=V=5$ and $A \neq 0$. The solid line is the RMT prediction (45).

It shows that in the limit of large number of channels there exist a strip in the complex $\kappa-$ plane which is free of resonances. This is in agreement with previous findings [27, 30, 44]. In the case of maximal coupling i.e. $g=1$, the power law (46) extends to infinity, leading to divergencies of the various moments of $\gamma$ 's. Using (45) we recover the well known Moldauer-Simonius relation [30] for the mean resonance width [37]

$$
\langle\gamma\rangle_{k}=-\frac{\sum_{i=1}^{V} \ln \left(\left|\left\langle S^{D}\right\rangle_{k}\right|^{2}\right)}{2 \pi}
$$

The resonance width distribution for a $V=5$ regular and generic graph is shown in figure $3 \mathrm{~b}$ together with the RMT prediction, which reproduces the numerical distribution quite well. Figure 4 shows a similar comparison for a Neumann graph. The relatively high abundance of resonances in the vicinity of the real axis conforms with the expectations.

\subsection{The Form Factor}

To investigate further the dynamical origin of the resonance fluctuations, we study the resonance two-point form factor $K(t)$. The main advantage of $K(t)$ is that it allows us to study the resonance fluctuations in terms of classical orbits. It is defined as

$$
K_{R}(t) \equiv \int \mathrm{d} \chi \mathrm{e}^{i 2 \pi \chi \mathcal{L} t} R_{2}(\chi) .
$$

where $t$ measures lengths in units of the Heisenberg length $l_{H}=\mathcal{L}$ and $R_{2}(\chi)$ is the excess probability density of finding two resonances at a distance $\chi$,

$$
R_{2}\left(\chi ; k_{0}\right)=\left\langle\tilde{d}_{R}\left(k+\frac{\chi}{2}\right) \tilde{d}_{R}\left(k-\frac{\chi}{2}\right)\right\rangle_{k}=\left\langle\frac{\Delta}{2 k_{0}} \int_{-k_{0}}^{k_{0}} \tilde{d}_{R}\left(k+\frac{\chi}{2}\right) \tilde{d}_{R}\left(k-\frac{\chi}{2}\right) d k\right\rangle_{k_{0}} \cdot(
$$




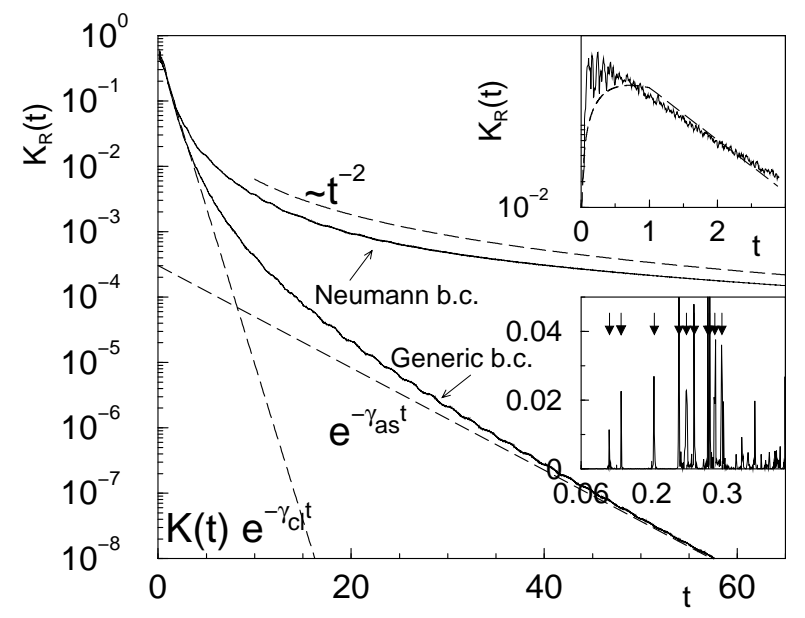

Figure 5. The form factors $K_{R}(t)$ for a complete $V=5$ graph, with either generic or Neumann boundary conditions, $A \neq 0$ and $M=V$. The data were averaged over 5000 spectral intervals and smoothed on small $t$ intervals. Upper inset: $K_{R}(t)$ for small times. Solid line correspond to the numerical data for the pentagon with generic boundary conditions while dashed line is the approximant $K_{R}(t) \approx K(t) \exp \left(-\gamma_{c l} t\right)$. Lower inset: $K_{R}(t)$ calculated with high resolution. The lengths of periodic orbits of the close graph are indicated by arrows.

Above $\langle\ldots\rangle_{k_{0}}$ indicate averaging oven a number of spectral intervals of size $\Delta_{k}=2 k_{0}$, centered around $k_{0}$. Here $\tilde{d}_{R}(k)$ is the oscillatory part of $d_{R}(k)$ (see Eq. (31)). Substituting the latter in Eqs. (48,49) we obtain $K_{R}(t)$ in terms of periodic orbits and their repetitions

$$
K_{R}(t)=\frac{2 \mathcal{N}}{\mathcal{L}^{2}}\left|\sum_{p} \sum_{r} l_{p} \tilde{\mathcal{A}}_{p}^{r} \mathrm{e}^{i\left(k l_{a}+\Theta_{a}\right)} \delta_{\mathcal{N}}\left(t-r l_{p} / \mathcal{L}\right)\right|^{2} .
$$

where $\delta_{\mathcal{N}}(x)=(\sin (\mathcal{N} x / 2)) /(\mathcal{N} x / 2)$ and $\mathcal{N}=\frac{\Delta_{k}}{\Delta}$. A similar sum contributes to the spectral form factor of the compact graph [13, 14]. However, the corresponding amplitudes are different due to the fact that $\tilde{\mathcal{A}}_{p}$ contains also the information about the escape of flux to the leads.

Assuming that all periodic orbits decay at the same rate, one would substitute $\tilde{\mathcal{A}}_{p}$ with $\mathcal{A}_{p} \exp \left(-n_{p} \gamma_{c l} / 2\right)$ where $\mathcal{A}_{p}$ is the weight assign to the periodic orbit of the corresponding close system. Then Eq. (50) takes the following simple form

$$
K_{R}(t) \approx K(t) \mathrm{e}^{-\gamma_{c l} t},
$$

where $K(t)$ is the form factor of the compact system [29]. Notice that for $t \ll \gamma_{c l}^{-1}$ the resonance form factor $K_{R}(t)$ is equal to $K(t)$. This is reasonable since an open system cannot be distinguished from a closed one during short times. This simple approximation is checked in the inset of figure 5 (see dashed line) and it is shown to 
reproduce the numerical data rather well in the domain $t \leq 5$. The asymptotic decay is dominated by the resonances which are nearest to the gap, and it cannot be captured by the crude argument presented above. For generic graph, $K_{R}$ decays exponentially but with a rate given by $\gamma_{a s}=\gamma_{g a p}$ (the best fit, indicated in figure 5 by the dashed line, give $\gamma_{a s}$ which agrees with $\gamma_{g a p}$ within $30 \%$ ). For the graph with Neumann boundary conditions, $\gamma_{\text {gap }}=0$ and one expects an asymptotic power-law decay. The corresponding best fit (see dashed line in figure 5) shows $t^{-2}$.

In Hamiltonian systems in more than one dimension, the size of the spectral interval $\Delta_{k}$ where the spectral average is performed is limited by the requirement that the smooth part of the spectral density is approximately constant. Here instead we can take arbitrarily large spectral intervals since the smooth spectral density is constant 13, 14. This way, one can reach the domain where the function $K_{R}(t)$ is composed of arbitrarily sharp spikes which resolve completely the length spectrum for lengths which are both smaller and larger than $l_{H}$. In the inset of figure 5 we show the numerical $K(t)$ calculated with high enough resolution. In the same inset we mark with arrows the location of the lengths of short periodic orbits. We notice that as long as $t \mathcal{L}$ is shorter than the length of the shortest periodic orbit, $K(t)=0$. With increasing $t$, the periodic orbits become exponentially dense and therefore the peaks start to overlap, giving rise to a quasi continuum described approximately by Eq. (51).

\subsection{Ericson fluctuations}

As was mentioned above, the scattering cross sections are dominated by either isolated resonances, or by overlapping resonances whose fluctuations follow a typical pattern. These patterns were first discussed by Ericson [45] in the frame of nuclear physics and were shown to be one of the main attributes of chaotic scattering [26]. The transition between the two regimes is controlled by the Ericson parameter (37). Typical fluctuating cross sections are shown in figure 2.

A convenient measure for Ericson fluctuations is the autocorrelation function

$$
C(\chi ; \nu)=\frac{1}{\Delta j} \sum_{j=j_{\min }}^{j_{\max }}\left\langle S_{j, j+\nu}^{(M)}\left(k+\frac{\chi}{2}\right) S_{j, j+\nu}^{(M) \star}\left(k-\frac{\chi}{2}\right)\right\rangle_{k}
$$

where $\Delta j=j_{\max }-j_{\min }+1$. To evaluate Eq. (52) we substitute the expression of the $S$-matrix from Eq. (14) and split the sum over trajectories into two distinct parts: the contributions of short trajectories are computed explicitly by following the multiple scattering expansion up to trajectories of length $l_{\text {max }}$. The contribution of longer orbits are approximated by using the diagonal approximation, which results in a Lorentzian with a width $\gamma_{E r}$. Including explicitly up to $n=3$ scattering events we get,

$$
\begin{aligned}
C(\chi ; \nu) & \approx G \mathrm{e}^{i l_{\max } \chi} \frac{\gamma_{E r}}{\gamma_{E r}-i \chi}+\frac{1}{\Delta j} \sum_{j=j_{\min }}^{j_{\max }}\left[\tau^{4} \mathrm{e}^{i \chi L_{j, j+\nu}}+\tau^{4} \rho^{4} \mathrm{e}^{3 i \chi L_{j, j+\nu}}\right. \\
& \left.+\tau^{6} \sum_{m \neq j, j+\nu} \mathrm{e}^{i \chi\left(L_{j, m}+L_{m, j+\nu}\right)}\right]
\end{aligned}
$$




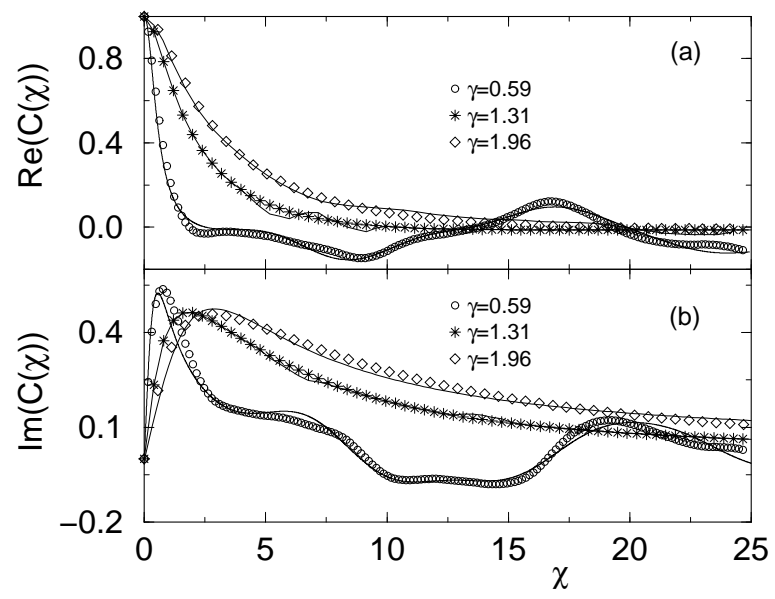

Figure 6. The autocorrelation function $C(\chi, \nu=1)$ for regular graphs with Neumann boundary conditions. (o) correspond to a graph with $\gamma=0.59$ (isolated resonance regime), $(\star)$ to a graph with $\gamma=1.36$ (intermediate regime) while $(\diamond)$ to a graph with $\gamma \simeq 2$ (overlapping resonances regime). The solid lines correspond to the theoretical expression (53): (a) The real part of $C(\chi, \nu=1)$; (b) The imaginary part of $C(\chi, \nu=1)$.

where $G$ is determined by the condition $C(\chi=0 ; \nu)=1$. The interplay between the contributions of long and short periodic orbits is shown in figure 6. For overlapping resonances, the autocorrelation function is well reproduced by a Lorentzian while for the case of isolated resonances one can clearly see the contributions of short paths.

In figure 7 we report the mean resonance width $\langle\gamma\rangle_{k}$ calculated numerically for various graphs, the parameter $\gamma_{\mathrm{Er}}$ extracted from the best fit of the numerical $C(\chi)$ with Eq. (53), together with the RMT prediction Eq. (47), and the classical expectation given by Eq. (38). The results justify the use of the classical estimate especially in the limit $V \rightarrow \infty$ for fixed $v / V$ (which is the analogue of the semiclassical limit). In this limit, the RMT and the classical estimate coincide.

\section{4. $S$-matrix statistics}

One can check further the applicability of RMT by studying the entire $S^{(M)}$-matrix distribution function. The probability density of $M \times M$ unitary $S^{(M)}$-matrices is defined in a $M+\beta M(M-1) / 2$ parameter space and was found first in [31 to be given by the Poisson Kernel

$$
d \mathcal{P}_{\bar{S}}\left(S^{(M)}\right)=p_{\bar{S}}(S) d \mu(S)=C_{\beta} \frac{\left[\operatorname{det}\left(1-\bar{S} \bar{S}^{\dagger}\right)\right]^{(\beta M+2-\beta) / 2}}{\left|\operatorname{det}\left(1-\bar{S} \bar{S}^{\dagger}\right)\right|^{(\beta M+2-\beta)}} d \mu_{\beta}(S)
$$




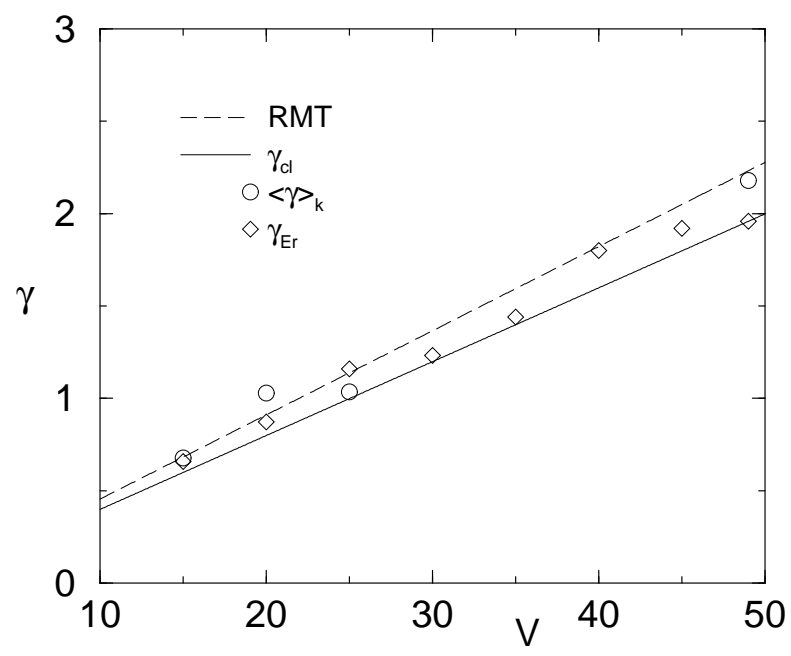

Figure 7. The mean resonance width $\langle\gamma\rangle_{k}$, autocorrelation width $\gamma_{E r}$, the classical expectation $\gamma_{c l}$, and the RMT prediction (47) vs. $V$ for various graphs with Neumann boundary conditions and constant valency $v=14$.

where $C_{\beta}$ is a normalization constant which depend on the symmetry class. All systemspecific relevant informations are included in the ensemble average $S^{(M)}$ - matrix, defined as $\bar{S}_{i, j}=\left\langle S^{D}\right\rangle_{k}$. For regular graphs, $\bar{S}$ is proportional to the unit matrix i.e. $\bar{S}_{i, j}=\frac{1-v}{1+v} \delta_{i, j}$, while the eigenvectors are distributed uniformly and independent from the eigenphases. The invariant measure $d \mu_{\beta}(S)$ is given as

$$
d \mu_{\beta}\left(S^{(M)}\right)=\prod_{i<j}\left|\mathrm{e}^{i \phi_{i}}-\mathrm{e}^{i \phi_{j}}\right|^{\beta} \prod_{i=1}^{M} d \phi_{i} d \Omega
$$

where $d \Omega$ is the solid angle on the $M$ dimensional unit hyper-sphere.

For large $M$ values, a numerical check of this probability distribution is prohibitive. However, for $M=2$ one can find easily the exact form of (54) and compare with the numerical results. The resulting distribution of the eigenphases $\phi_{1}, \phi_{2}$ of the $2 \times 2$ scattering matrices of regular graphs is given by

$$
\begin{aligned}
& d \mathcal{P}_{\bar{S}}\left(S^{(2)}\right)= \\
= & \frac{C_{\beta}\left(\frac{4 v}{(v+1)^{2}}\right)^{\beta+2} \sin ^{\beta}\left(\frac{\omega}{2}\right)}{\left[1-4 \bar{S} \cos \left(\frac{\omega}{2}\right) \cos \psi+2 \bar{S}^{2}\left(2 \cos ^{2}\left(\frac{\omega}{2}\right)+\cos (2 \psi)\right)-4 \bar{S}^{3} \cos \psi \cos \left(\frac{\omega}{2}\right)+\bar{S}^{4}\right]^{\frac{\beta+2}{2}}} d \omega d \psi
\end{aligned}
$$

where we used the notation $\psi=\frac{\phi_{1}+\phi_{2}}{2}$ and $\omega=\phi_{1}-\phi_{2}$. Integrating Eq. (56) over $\omega$, $\psi$ we get the corresponding distribution functions $\mathcal{P}_{\bar{S}}(\omega)$ and $\mathcal{P}_{\bar{S}}(\psi)$. Our numerical results for an ensample of $S$-matrices calculated for different realizations of the lengths of the graphs are reported in figure 8 together with the RMT predictions (56) for a regular graph with two channels. An overall good agreement is seen both for $A=0$ and $A \neq 0$. 


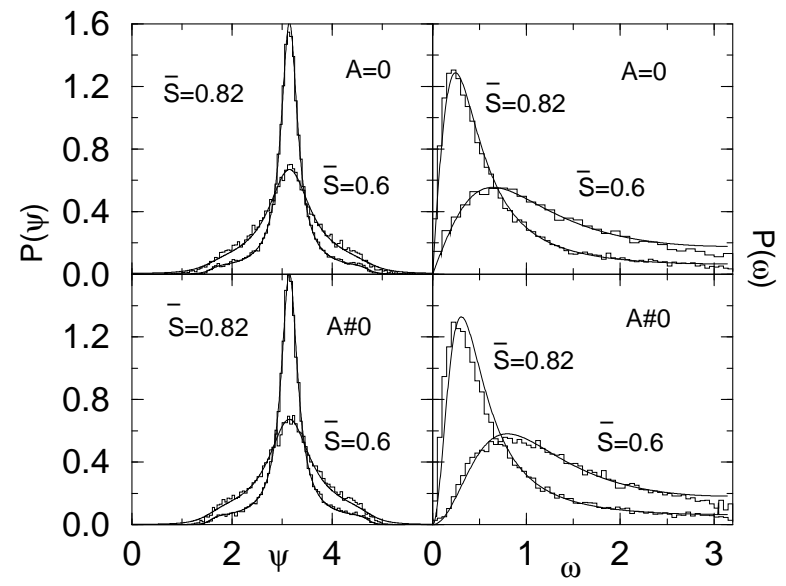

Figure 8. The $\mathcal{P}(\psi)$ and $\mathcal{P}(\omega)$ distributions for a $2 \times 2$ scattering matrix $S$. The solid lines correspond to the predictions of RMT (56). The upper panel correspond to $A=0$ while the lower panel to $A \neq 0$.

\subsection{Partial delay times statistics}

The Wigner delay time captures the time-dependent aspects of quantum scattering. It can be interpreted as the typical time an almost monochromatic wave packet remains in the interaction region. It is defined as

$$
T_{W}=\frac{1}{2 i M} \operatorname{tr}\left[S^{(M) \dagger} \frac{d S^{(M)}(k)}{d k}\right]=\frac{1}{2 M} \sum_{i=1}^{M} \frac{\partial \phi_{i}(k)}{\partial k},
$$

where $\phi_{i}$ are the eigen-phases of the $S^{(M)}$-matrix. The partial derivatives $\frac{\partial \phi_{i}(k)}{\partial k}$ are the partial delay times and their statistical properties were studied extensively within the RMT [35, 37, 39]. For the one-channel case it was found [37, 39] that the probability distribution of the scaled (with the mean level spacing $\Delta$ ) partial delay times $T_{i}=\frac{\Delta}{2 \pi} \frac{\partial \phi_{i}(k)}{\partial k}$ is

$$
\mathcal{P}_{\bar{S}}^{(\beta)}(T)=\frac{\left(\frac{\beta}{2}\right)^{\beta / 2}}{\Gamma\left(\frac{\beta}{2}\right) T^{2+\beta / 2}} \int_{0}^{2 \pi} \mathcal{P}(\phi)^{1+\beta / 2} \mathrm{e}^{-\frac{\beta}{2 T} \mathcal{P}(\phi)} d \phi
$$

where $\mathcal{P}(\phi)$ is the Poisson Kernel Eq. (54). The general case of $M$-equivalent open channels was studied in [35, 37] where it was found that for broken time reversal symmetry the probability distribution of partial delay times is

$$
\mathcal{P}(T)=\frac{(-1)^{M}}{M ! T^{M+2}} \frac{\partial^{M}}{\partial\left(T^{-1}\right)^{M}}\left[\mathrm{e}^{-g / T} I_{0}\left(T^{-1} \sqrt{\left.\left(g^{2}-1\right)\right)}\right]\right.
$$

where $I_{0}(x)$ stands for the modified Bessel function.

To investigate the statistical properties of the partial delay times for our system we had calculated $\mathcal{P}(T)$ for various graphs. The resulting distributions are shown in figure 9 together with the RMT predictions (58,59). An overall agreement is evident. 

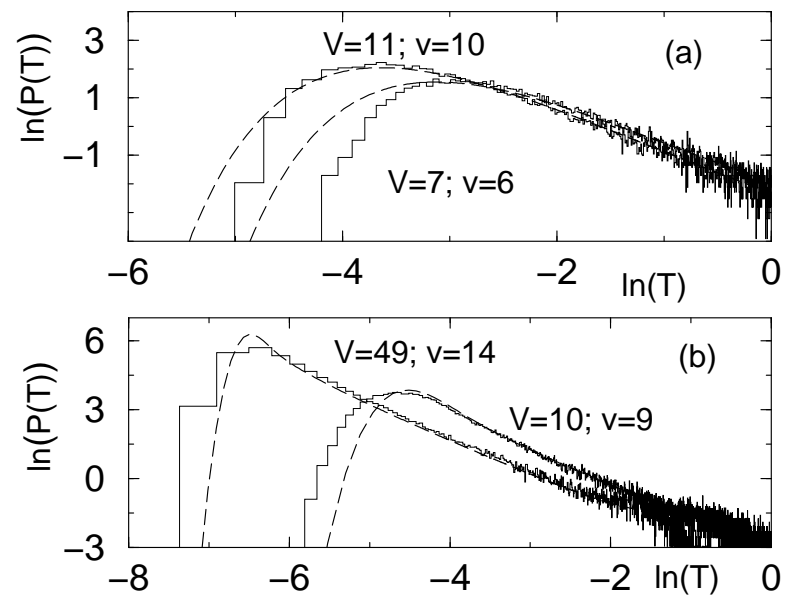

Figure 9. The distribution of the scaled partial delay times $T$ for various graphs with Neumann boundary conditions. The dashed lines correspond to the RMT expectation (58) 59): (a) One channel and $A=0$; (b) $M=V$ channels and $A \neq 0$.

Deviations appear at the short time regime (i.e. short orbits), during which the "chaotic" component due to multiple scattering is not yet fully developed [28].

\subsection{Conductance Distribution}

Due to the recent experimental investigation of electronic transport through mesoscopic devices [46], the study of the statistical properties of the conductance gained some importance. For a device connected to reservoirs by leads, the Landauer- Büttiker formula relates its conductance $G$ to the transmission coefficient $T_{G}$ by the expression $G=\left(2 \mathrm{e}^{2} / \hbar\right) T_{G}$. When each lead supports one channel, the transmission coefficient can be written in terms of the $S^{(M)}$-matrix as

$$
T_{G}=\sum_{i \neq j}^{M}\left|S_{i, j}^{(M)}\right|^{2}=1-\left|S_{j, j}^{(M)}\right|^{2}
$$

where $j$ is the input channel.

In the absence of direct processes, the distribution of conductance $\mathcal{P}\left(T_{G}\right)$ for arbitrarily number of channels was worked out within RMT, and the results describe in a satisfactory way both the numerical calculations and the experimental data (for a review see [40] and references therein). However in cases where direct processes appear significantly, one must use the Poisson's kernel (54) in its full generality. This is exactly the case with Neumann graphs since $\bar{S} \neq 0$. The probability distribution of the transmission $T_{G}$ is then

$$
\mathcal{P}\left(T_{G}\right)=\int \delta\left(T_{G}-\sum_{i \neq j}\left|S_{i, j}\right|^{2}\right) \mathcal{P}_{\bar{S}}(S) d \mu(S) .
$$




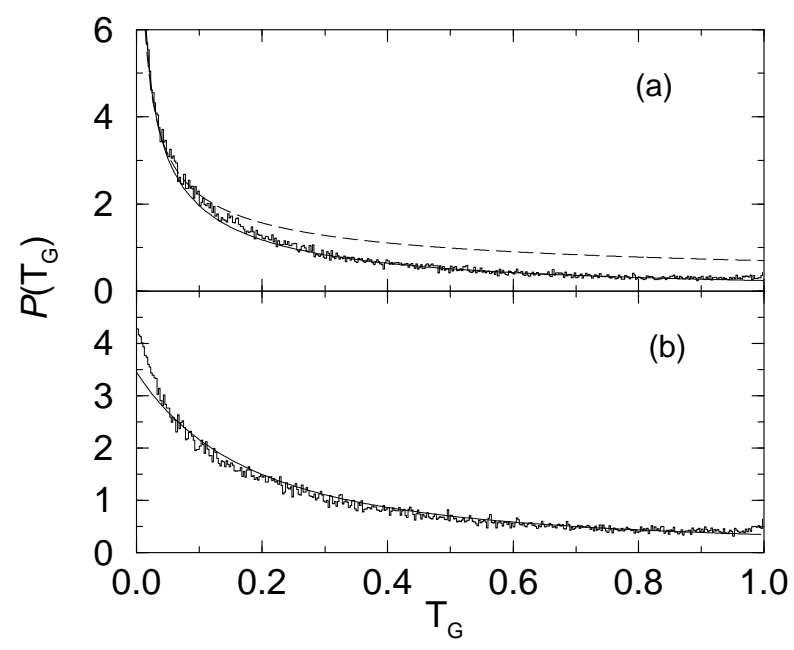

Figure 10. Conductance distribution $\mathcal{P}\left(T_{G}\right)$ for a graph with $M=2$ channels: (a) Time reversal symmetry is preserved $(A=0)$. The solid line is the RMT results Eq. (61) where we had used Eq. (56) for the Poisson Kernel while the dashed line is the approximate expression (62); (b) Broken time reversal symmetry $(A \neq 0)$. The solid line is the RMT result Eq. (63).

For the case $M=2$ and for a diagonal $\bar{S}$-matrix (only direct reflection) with equivalent channels $\bar{S}_{11}=\bar{S}_{22}=\bar{S}$, Eq. (61) can be worked out analytically [34] in the limit of strong reflection and $T_{G} \ll 1$. The resulting expression is:

$$
\mathcal{P}_{\beta=1}\left(T_{G}\right)=\frac{8}{\pi^{2}\left(1-\bar{S}^{2}\right)} T_{G}^{-1 / 2} \quad, \quad T_{G} \ll\left(1-\bar{S}^{2}\right)^{2}
$$

For $\beta=2$ one can compute in a close form the whole distribution [40]

$$
\mathcal{P}_{\beta=2}\left(T_{G}\right)=\left(1-\bar{S}^{2}\right) \frac{\left(1-\bar{S}^{4}\right)^{2}+2 \bar{S}^{2}\left(1+\bar{S}^{4}\right) T_{G}+4 \bar{S}^{4} T_{G}^{2}}{\left(\left(1-\bar{S}^{2}\right)^{2}+4 \bar{S}^{2} T_{G}\right)^{5 / 2}} .
$$

Our numerical results for a regular graph with two leads are plotted in figure 10 together with the RMT results (61,662,63). Notice that, the small conductances are emphasized, because of the presence of direct reflection and no direct transmission. At the same time, the most pronounced differences between the two symmetry classes are

for $T_{G} \ll 1$, where for $\beta=1$ the conductance distribution diverges while it take a finite value for $\beta=2$.

\section{Scattering from star-graphs.}

So far, we have studied scattering from well connected graphs, and we have shown that many statistical properties of the scattering matrix are described by RMT. We shall dedicate this section to demonstrate the statistical properties of the $S$-matrix for graphs which have non-uniform connectivity. 
A representative example of this category are the "star" (or "Hydra") graphs [14, 16]. They consist of $v_{0}$ bonds, all of which emanate from a single common vertex labeled with the index $i=0$. The vertex at $i=0$, will be referred to in the sequel as the head. The total number of vertices for such a graph is $V=v_{0}+1$, and the vertices at the end of the bonds will be labeled by $i=1 \cdots v_{0}$. The star is a bipartite graph, a property which implies e.g., that there exists no periodic orbits of odd period [14]. To turn a star graph into a scattering system we add a lead to its head.

It is a simple matter to derive the scattering matrix $S=S^{(M=1)}$ for a Neumann star. It reduces to a phase factor,

$$
S(k) \equiv \mathrm{e}^{i \phi(k)}=\frac{-\sum_{i=1}^{v_{0}} \tan \left(k L_{i}\right)+i}{\sum_{i=1}^{v_{0}} \tan \left(k L_{i}\right)+i}
$$

The spectrum $\left\{k_{n}\right\}$ of the close system can be identified as the set of wave-vectors for which $S(k)$ equals 1 , which implies that no current flows in the lead. The resulting quantization condition is

$$
1-S(k)=0 \longleftrightarrow \frac{2 \sum_{i=1}^{v_{0}} \tan \left(k_{n} L_{i}\right)}{\sum_{i=1}^{v_{0}} \tan \left(k L_{i}\right)+i}=0,
$$

which is satisfied once $\sum_{i=1}^{v_{0}} \tan \left(k_{n} L_{i}\right)=0$. This is identical with the condition derived in 14 .

The poles $\left\{\kappa_{n}\right\}$ are the complex zeros of

$$
\sum_{i=1}^{v_{0}} \tan \left(\kappa_{n} L_{i}\right)+i=0
$$

To first order in $\frac{1}{v_{0}}$, we get

$$
\Gamma_{n}^{(1)}=\frac{1}{\sum_{i=1}^{v_{0}} \frac{L_{i}}{\cos \left(2 k_{n} L_{i}\right)+1}}
$$

which can be used as a starting point for the exact evaluation of the poles. For the latter one has to perform a self consistent search for the complex zeros of the secular equation (66). This is a time consuming process and the correct choice of the initial conditions is very important.

In figure 11 we present our numerical results for the distribution of rescaled resonance widths $\mathcal{P}(\gamma)$ for a star with $v_{0}=20$. The data are in excellent agreement with the RMT expectation given in Eq. (44). We point that in this case the coupling to the continuum is weak since $g \simeq 10 \gg 1$ and therefore the $\chi^{2}$-distribution with $M=1$ is applicable.

Using Eq. (64) we get the following relation for the scaled delay times

$$
T(k)=\frac{\Delta}{2 \pi} \frac{2 \sum_{i=1}^{v_{0}} \frac{L_{i}}{\cos ^{2}\left(k L_{i}\right)}}{1+\left(\sum_{i=1}^{v_{0}} \tan k L_{i}\right)^{2}}
$$

which can be used to generate $\mathcal{P}(T)$. The latter is reported in figure 12 together with the RMT prediction (59). We notice that although the tail of the distribution agrees reasonably well with the RMT prediction, there are considerable deviations at the origin. 
Quantum Graphs: A simple model for Chaotic Scattering

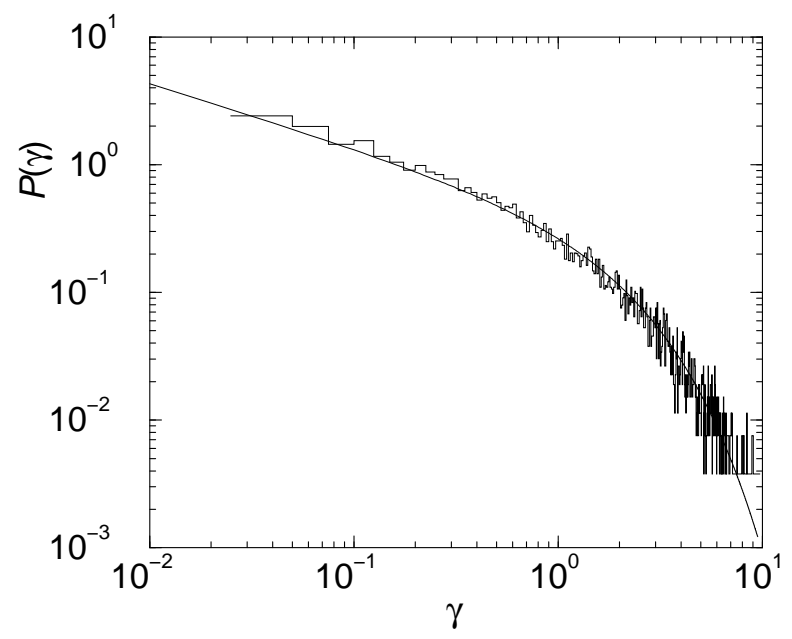

Figure 11. The rescaled resonance width distribution $\mathcal{P}(\gamma)$ for a star graph with $v_{0}=20$. The solid line is the RMT prediction Eq. (44).

A peculiarity of the star graph is that the mean time delay is twice larger than the expected one from semiclassical considerations. To be more specific, for a generic chaotic system coupled to $M$ channels, one has [41]

$$
\langle T\rangle_{k} \simeq \frac{M+1}{M} \frac{\Delta}{2 \pi}\left\langle l_{p}\right\rangle_{k}
$$

where $\left\langle l_{p}\right\rangle_{k} \simeq \Gamma_{c l}^{-1}$ is the average length of the classical paths inside the interaction area. Thus for the star with $M=1$, we would expect based on Eq. (69) that $\langle T\rangle_{k} \simeq 2 \Gamma_{c l}$. However, due to the fact that all the periodic orbits on the star graph are self tracing we get an additional factor of 2 and thus

$$
\langle T\rangle_{k}=\frac{\Delta}{2 \pi} 4 \Gamma_{c l}^{-1} .
$$

The corresponding classical decay rate $\Gamma_{c l}$ can be found exactly by a direct evaluation of the eigenvalues of the classical evolution operator $\tilde{U}$. One can easily show that

$$
\tilde{U}=\left(\begin{array}{ll}
0 & \mathbf{1} \\
\left(\tilde{\sigma}^{(0)}\right)^{2} & 0
\end{array}\right)
$$

where $\tilde{\sigma}^{(0)}$ is the $v_{0} \times v_{0}$ vertex scattering matrix at the star head as defined in Eq. (5), while 1 denotes the $v_{0} \times v_{0}$ identity matrix. The square of the classical evolution operator $\tilde{U}^{2}$ has a block diagonal form

$$
\tilde{U}^{2}=\left(\begin{array}{ll}
\left(\tilde{\sigma}^{(0)}\right)^{2} & 0 \\
0 & \left(\tilde{\sigma}^{(0)}\right)^{2}
\end{array}\right) .
$$

and its spectrum consists of the values $1-\frac{4}{\left(1+v_{0}\right)^{2}}$ with multiplicity 2 and $1-\frac{4}{\left(1+v_{0}\right)}$ with 


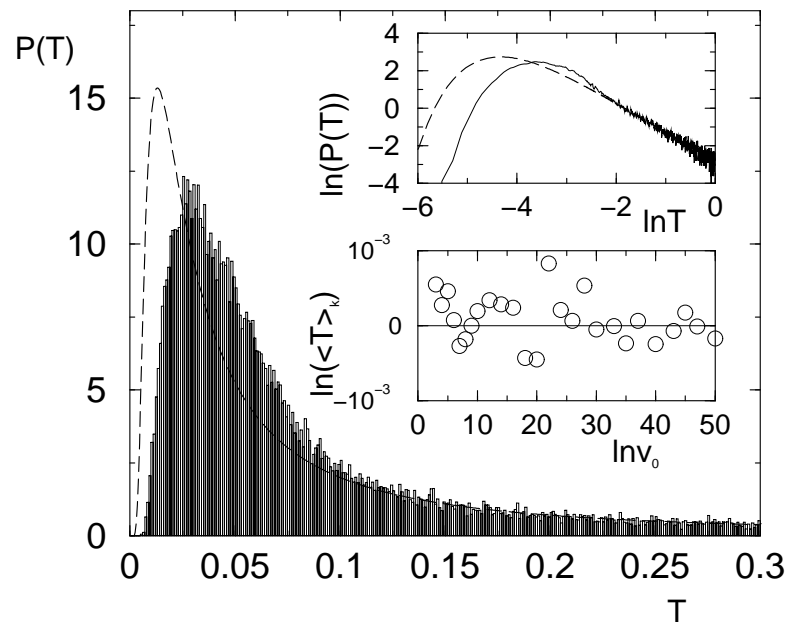

Figure 12. The distribution of the scaled partial delay times $\mathcal{P}(T)$ for a star graph with $v_{0}=20$. The dashed line is the RMT prediction Eq. (58). In the upper inset we show the same data in a double logarithmic plot. In the lower inset we plot the numerical results for $\langle T\rangle_{k}(\circ)$. The solid line is the asymptotic value 1 expected from semiclassical considerations.

multiplicity $2 v_{0}-2$. Therefore the spectrum of $\tilde{U}$ is

$$
\begin{aligned}
\lambda_{u} & = \pm \sqrt{1-\frac{4}{\left(1+v_{0}\right)^{2}}} \\
& = \pm \sqrt{1-\frac{4}{1+v_{0}}} \quad \text { with multiplicity } v_{0}-1 .
\end{aligned}
$$

For short times where the classical evolution is applicable, the dominant eigenvalue is $\lambda_{u}=\sqrt{1-\frac{4}{1+v_{0}}}$ leading to a classical decay rate

$$
\Gamma_{c l} \simeq \frac{2}{1+v_{0}}
$$

Substituting Eq. (74) we get eventually that $\langle T\rangle_{k}=\frac{1+v_{0}}{v_{0}} \overrightarrow{v_{0} \rightarrow \infty} 1$ which indicate that the mean time a particle spend inside the interaction regime is proportional to the Heisenberg time. Our numerical calculations reported in the lower inset of Fig. 12 agrees nicely with the semiclassical prediction (70).

Finally, we analyze the distribution of $S$ when generated over different realizations of the lengths of the bond. For the one channel case this is equivalent with the distribution $\mathcal{P}(\phi)$ of the phase of the $S$-matrix. To derive the latter, it is convenient to rewrite the $S$-matrix in the following form

$$
S \equiv \exp (i \phi)=(-K+i) /(K+i)
$$




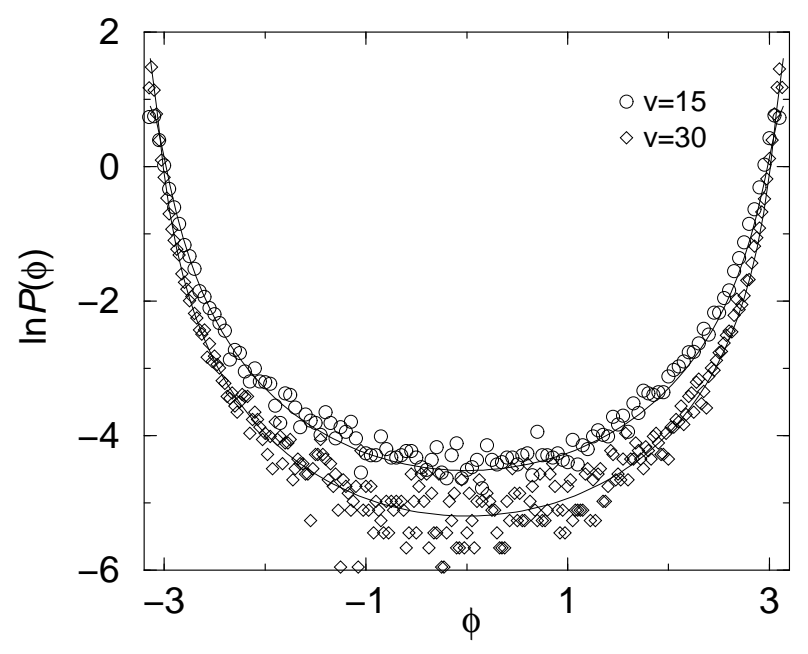

Figure 13. The distribution of phases $\mathcal{P}(\phi)$ of the $S_{H}$-matrix for two stars with $v_{0}=15$ and $v_{0}=30$. The solid lines are the corresponding theoretical predictions (77).

with $K=\sum_{i=1}^{v} \tan \left(k L_{i}\right)$. The probability distribution of $K$ is

$$
\begin{aligned}
\mathcal{P}(K) & =\left\langle\delta\left(K-\sum_{i=1}^{v} \tan \left(k L_{i}\right)\right)\right\rangle_{L} \\
& =\frac{1}{2 \pi} \int \mathrm{e}^{i K x} d x\left(\frac{1}{\Delta L} \int_{L_{\min }}^{L_{\max }} d L \mathrm{e}^{-i x \tan (k L)}\right)^{v} \\
& =\frac{1}{\pi} \frac{v}{v^{2}+K^{2}} .
\end{aligned}
$$

Thus, with $\bar{S} \equiv\langle S\rangle=\frac{1-v}{1+v}$, we get

$$
\mathcal{P}(\phi)=\mathcal{P}(K)\left|\frac{d K}{d \phi}\right|=\frac{1}{2 \pi} \frac{1-\bar{S}^{2}}{1+\bar{S}^{2}-2 \bar{S} \cos \phi} .
$$

Equation (77) reproduces the Poisson's kernel for a one-channel scattering matrix, derived in the framework of RMT [37]. The conditions under which this result is derived in [37] are fulfilled exactly in the present case. Our numerical results are reported in figure 13 and are in excellent agreement with the theoretical prediction Eq. (77).

\section{Conclusions}

In this paper, we turned quantum graphs into scattering systems. We show that they combine the desirable features of both behaving "typically" and being mathematically simple. Thus, we propose them as a convenient tool to study generic behavior of chaotic scattering systems. 
The classical dynamics on an open graph was defined, and the classical staying probability was shown to decay in an exponential way. The resulting classical escape rate was calculated and used to describe the properties of the corresponding quantum system. The scattering matrix was written in terms of classical orbits and an exact trace formula for the resonance density was found. A gap for the resonance widths has been obtained for "generic" graphs and its absence was explained for Neumann graphs. An analysis of the cross section autocorrelation function was performed and its non-universal characteristics were explained in terms of the short classical scattering trajectories. Finally, due to the relative ease by which a large number of numerical data can be computed for the graph models, we had performed a detail statistical analysis of delay times, resonance widths and distribution of the $S$-matrix. Our results compares well with the predictions of RMT indicating that our model can be used in order to understand the origin of the connection between RMT and the underlying classical chaotic dynamics.

The results reported here, complete our previous investigations on graphs. We conclude that quantum graphs may serve as a convenient paradigm in the area of quantum chaos, both for spectral and scattering studies.

\section{Acknowledgments}

We acknowledge many useful discussions with Y. Fyodorov and H. Schanz. This work was supported by the Minerva Center for Nonlinear Physics, and an Israel Science

Foundation Grant. (T. K.) acknowledges a postdoctoral fellowship from the Feinberg School, The Weizmann Institute of Science.

[1] S. Alexander, Phys. Rev. B 27, 1541 (1985).

[2] C. Flesia, R. Johnston and H. Kunz, Europhys. Lett. 3 , 497 (1987).

[3] R. Mitra and S. W. Lee, Analytical techniques in the theory of guided waves, Macmillan, New York.

[4] P. W. Anderson, Phys. Rev. B 23, 4828 (1981); B. Shapiro, Phys. Rev. Lett. 48823 (1982).

[5] J. T. Chalker and P. D. Coddington, J. Phys. C 21, 2665 (1988); Rochus Klesse and Marcus Metzler, Phys. Rev. Lett. 79, 721 (1997); R. Klesse, Ph. D. Thesis, Universitat zu Köln, AWOSVerlag, Erfurt, 1996.

[6] Y. Avishai, J. M. Luck, Phys. Rev. B 45, 1074 (1992); T. Nakayama, K. Yakubo, R. L. Orbach, Rev. Mod. Phys. 66, 381 (1994).

[7] Y. Imry, Introduction to Mesoscopic Systems, Oxford, New York (1996); D. Kowal, U. Sivan, O. Entin-Wohlman, Y. Imry, Phys. Rev. B 42, 9009 (1990).

[8] J. Vidal, G. Montambaux, D. Doucot, Phys. Rev. B 62, R16294 (2000).

[9] Zhang et al. Phys. Rev. Lett. 81, 5540 (1998). 
[10] Colin de Verdière. Spectres de Graphes. Société Mathématique de France, Marseille (1998).

[11] P. Exner, Phys. Rev. Lett. 74, 3503 (1995); P. Exner, P. Seba, Rep. Math. Phys. 28, 7 (1989); J. E. Avron, P. Exner, Y. Last, Phys. Rev. Lett. 72, 896 (1994); R. Carlson, Electronic Journal of Differential Equations 6, 1 (1998).

[12] Jean-Pierre Roth, in: Lectures Notes in Mathematics: Theorie du Potentiel, A. Dold and B. Eckmann, eds. (Springer-Verlag) 521-539.

[13] Tsampikos Kottos and Uzy Smilansky. Phys. Rev. Lett. 79, 4794 (1997).

[14] Tsampikos Kottos and Uzy Smilansky, Annals of Physics 274, 76 (1999).

[15] Akkermans E, Comtet A, Desbois J, Montambaux G, Texier C, Annals of Physics 284, 10 (2000).

[16] Berkolaiko G. and Keating J. Phys. A 327827 (1999); Berkolaiko G., Bogomolny E.B., and Keating J. Phys. A 34335 (2001).

[17] H. Schanz and U. Smilansky. Phys. Rev. Lett. 84, 1427 (2000); Proceedings of the Australian Summer School on Quantum Chaos and Mesoscopics, Canberra, Australia, 1999. chaodyn/9904007]; Tsampikos Kottos and Holger Schanz, Physica E 9, 523 (2001).

[18] G. Tanner, J. Phys. A 33, 3567 (2001).

[19] G. Berkolaiko, H. Schanz, R. S. Whitney, Phys. Rev. Lett. 88, 104101 (2002).

[20] F. Barra, P. Gaspard, J. Stat. Phys. 101, 283 (2000); F. Barra, P. Gaspard, Phys. Rev. E 63, $066215(2001)$.

[21] L. Kaplan, Phys. Rev. E 64, 036225 (2001); Tsampikos Kottos in preparation (2002).

[22] Tsampikos Kottos and Uzy Smilansky, Phys. Rev. Lett. 85, 968 (2000).

[23] F. Barra and P. Gaspard, Phys. Rev. E, 65, 016205 (2002).

[24] C. Texier and G. Montambaux, J. Phys. A 3410307 (2001); C. Texier, J. Phys. A 353389 (2002).

[25] W. H. Miller, Adv. Chem. Phys. 2569 (1974).

[26] U. Smilansky, in Les Houches Summer School on Chaos and Quantum Physics, M.-J. Giannoni et.al., eds. (North-Holland) 371-441 (1989) (see also, Les Houches Summer School on mesoscopic quantum physics, E. Akkermans et. al., eds. (North-Holland) 373-433 (1994)).

[27] P. Gaspard, in "Quantum Chaos", Proceedings of E. Fermi Summer School 1991, G. Casati et. al., eds. (North-Holland) 307; P. Gaspard and S. A. Rice, J. Chem Phys 90, 2225,2242,2255(1989); 91 E3279, (1989).

[28] R. Blümel and U. Smilansky, Phys. Rev. Lett. 64, 241 (1990).

[29] B. Eckhardt, I. Varga, P. Pollner, Physica E 9, 535 (2001).

[30] P. A. Moldauer, Phys. Rev. C 11, 426 (1975); P. A. Moldauer, Phys. Rep. 157, 907 (1967); M. Simonius, Phys. Lett. 52B, 279 (1974).

[31] P. Mello, P. Pereyra, and T. H. Seligman, Ann. Phys. (NY) 161, 254 (1985). 
[32] R. A. Jalabert, A. D. Stone and Y. Alhassid, Phys. Rev. Lett. 68, 3468 (1992).

[33] F. Haake, F. Izrailev, N. Lehmann, D. Saher, H-J Sommers, Z. Phys. B 88, 359 (1992); N. Lehmann, D. Saher, V. Sokolov, H-J Sommers, Nucl. Phys. A 582, 223 (1995).

[34] P. W. Brouwer and C. W. J. Beenakker, Phys. Rev. B 50, 11263 (1994).

[35] Y. V. Fyodorov and H.-J. Sommers, Phys. Rev. Lett. 76, 4709 (1996); Y. V. Fyodorov, D. V. Savin, and H.-J. Sommers, Phys. Rev. E 55 R4857 (1997).

[36] H-J Sommers, Y. V. Fyodorov, and M. Titov, J. Phys. A: Math. Gen. 32 L77 (1999); Y. Fyodorov, H-J Sommers, Pis'ma Zh. Eksp. Teor. Fiz. 63970 (1996) [JETP Lett. 63,1026 (1996)]

[37] Y. Fyodorov, H-J Sommers, J. Math. Phys. 381918 (1997); P. Mello in Les Houches Summer School on Chaos and Quantum Physics, E. Akkermans et.al., eds (North-Holland) 437-491 (1994).

[38] P. W. Brouwer, K. M. Frahm, C. W. Beenakker, Phys. Rev. Lett. 78, 4737 (1997).

[39] V. A. Gopar and P. Mello, Europhys. Lett. 42, 131 (1998).

[40] P. A. Mello and H. U. Baranger, Waves in Random Media 9, 105 (1999).

[41] E. Doron, and U. Smilansky, Nonlinearity 5, 1055 (1992); E. Doron, and U. Smilansky, Phys. Rev. Lett. 68, 1255 (1992).

[42] C. Mahaux and H. A. Weidenmuller, "Shell Model Approach in Nuclear Reactions", (NorthHolland, Amsterdam), (1969).

[43] M. Sh. Birman and D. R. Yafaev. The spectral shift function. The work of M. G. Krein and its further development. St. Petersburg Math. J., 4:833-870, 1993.

[44] V. Sokolov and G. Zelevinsky, Phys. Lett. B 20210 (1988); E. Sobeslavsky, F. M. Dittes, and I. Rotter, J. Phys. A: Math. Gen. 28, 2963 (1995).

[45] T. Ericson, Phys. Rev. Lett. 5, 430 (1960); T. Ericson, Ann. Phys. (NY) 23390 (1963).

[46] C. M. Marcus, A. J. Rimberg, R. M. Westervelt, P. F. Hopkins, and A. C. Gossard, Phys. Rev. Lett. 69, 506 (1992). 